\title{
A parallel subgradient projection algorithm for quasiconvex equilibrium problems under the intersection of convex sets*
}

\author{
Le Hai Yen and Le Dung Muu \\ Institute of Mathematics and TIMAS, Thang Long University
}

Email. lhyen@math.ac.vn; ldmuu@math.ac.vn

\begin{abstract}
In this paper, we studied the equilibrium problem where the bi-function may be quasiconvex with respect to the second variable and the feasible set is the intersection of a finite number of convex sets. We propose a projection-algorithm, where the projection can be computed independently onto each component set. The convergence of the algorithm is investigated and numerical examples for a variational inequality problem involving affine fractional operator are provided to demonstrate the behavior of the algorithm.
\end{abstract}

Keywords: Equilibria; Quasiconvexity; Intersection; Subgradient method; Projection method.

\section{Introduction}

Let $C$ be a nonempty closed convex set in $\mathbb{R}^{n}$ and $f: \mathbb{R}^{n} \rightarrow \mathbb{R} \cup\{+\infty\}$ be a given bifunction such that $f(x, y)<+\infty$ for every $x, y \in C$. We consider the problem

$$
\text { Find } x^{*} \in C: f\left(x^{*}, y\right) \geq 0 \quad \forall y \in C \text {. }
$$

This inequality is often called equilibrium problem. The interest of this problem is that it unifies many important problems such as the Kakutani fixed point, variational inequality, optimization and the Nash equilibrium problems 2, 3, 4, 15] in a convenient way. The inequality in (EP) first was used in [17] by Nikaido and Isoda for a convex game model. The first result for solution existence of (EP) has been obtained by Ky Fan in [7, where the bifunction $f$ can be quasiconvex with respect to the second argument. Suppose, as usual, that

${ }^{*}$ This work is supported by the NAFOSTED, Grant 101.01-2020.06. 
$f(x, x)=0$ for all $x \in C$, then it is easy to see that (EP) is equivalent to the fixed point problem

$$
\text { Find } x^{*} \in C: x^{*} \in S\left(x^{*}\right) \text {, }
$$

where the fixed point mapping $S$ is defined by taking

$$
S(x):=\operatorname{argmin}\{f(x, y): y \in C\} .
$$

This fact suggests the use of the iterative scheme $x^{k+1} \in S\left(x^{k}\right)$ for the fixed point problem to solve inequality $(E P)$. The first difficulty that we have to face with here is that the mapping $S$ may not be singleton, even it is not defined at every point of $C$, i.e., Problem $P(x)$ is not solvable. To overcome this difficulty one can use the auxiliary problem principle that states that if $f(x,$.$) is convex,$ subdifferentiable on $C$, then for any $r>0$, Problem $(E P)$ is equivalent to the following one

$$
\text { Find } x^{*} \in C: f\left(x^{*}, y\right)+r\left\|y-x^{*}\right\|^{2} \geq 0 \quad \forall y \in C,
$$

in the sense that their solution-sets coincide. Then the corresponding fixed point mapping takes the form

$$
s(x):=\operatorname{argmin}\left\{f(x, y)+r\|y-x\|^{2}: y \in C\right\} .
$$

Since $f(x,$.$) is convex and \|.-x\|^{2}$ is strongly convex, the latter mathematical program is always uniquely solvable. However in the case $f(x,$.$) is quasiconvex$ rather than convex, the auxiliary problem principle cannot be applied because of the fact that the mathematical programming problem defining the mapping $s($.$) is nonlonger convex, even not quasiconvex, and therefore solving it is an$ extremely difficult task. Based upon the auxiliary problem principle, a lot numbers of algorithms using techniques of mathematical programming methods have been developed for solving problem (EP), e.g. [3, 12, 16, 19, 22, 23, 24, 25] and the references therein, however to our best knowledges, all of them require that the bifunction $f$ is convex with respect to its second variable. In our recent preprint 26] we developed an algorithm for problem (EP), where the bifunction $f$ may be quasiconvex in its second variable. In order to handle the quasiconvexity, we used the metric projection onto $C$ along the direction defined by a star-subgradient of the quasiconvex function $f(x,$.$) , rather than solving$ the mathematical programming problem (10). However in general, the projection onto $C$ is not easy to compute. In this paper we continue our work by considering problem (EP) where the feasible set $C$ is the intersection of a finite number of convex sets (often in practice), and we propose a projection-algorithm, where the projection can be computed independently onto each component set.

The organization of this paper as the following. The next section are preliminaries on the quasiconvex function on $\mathbb{R}^{n}$ and its star-subdifferential. A parallel algorithm for solving (EP) when $C$ is the intersection of a finite number of convex sets is proposed, and its convergence analysis is studied in Section 3. In the last section, numerical experiences are provided to prove the efficiency of the algorithm for a class of equilibrium problems involving quasiconvex bifunctions. 


\section{Preliminaries on Quasiconvex Function and Its Subdifferentials}

First of all, let us recall the well known definitions on the quasiconvex function and its star- subdifferential that will be used to the algorithm.

Definition 2.1. 14 A function $\varphi: \mathbb{R}^{n} \rightarrow \mathbb{R} \cup\{+\infty\}$ is called quasiconvex on a convex subset $Y$ of $\mathbb{R}^{n}$ if and only if for every $x, y \in Y$ and $\lambda \in[0,1]$, one has

$$
\varphi[(1-\lambda) x+\lambda y] \leq \max [\varphi(x), \varphi(y)] .
$$

It is easy to see that $\varphi$ is quasiconvex on a convex set $Y$ if and only if the level set $\{x \in Y: \quad \varphi(x)<\alpha\}$ on $Y$ of $\varphi$ at $x$ is convex for every $\alpha \in \mathbb{R}$.

We recall that a function $\varphi: \mathbb{R}^{n} \rightarrow \mathbb{R}$ is said to be Lipschitz on $Y$ at a point $y \in Y$, if there exist a finite number $L>0$ such that

$$
|\varphi(x)-\varphi(y)| \leq L\|x-y\| \forall x \in Y .
$$

The star-sudifferential of $\varphi$, see e,g. [20] is defined as

$$
\partial^{*} \varphi(x):=\left\{g \in \mathbb{R}^{n}:\langle g, y-x\rangle<0 \forall y \in L_{\varphi}(x)\right\},
$$

where $L_{\varphi}(x):=\left\{y \in R^{n}: \varphi(y)<\varphi(x)\right\}$ is the level set of $\varphi$ at the level $\varphi(x)$. Clearly, if $\bar{L}_{\varphi}(x)$ is the closure of $L_{\varphi}(x)$, then

$$
\partial^{*} \varphi(x):=\left\{g \in \mathbb{R}^{n} ;\langle g, y-x\rangle \leq 0 \forall y \in \bar{L}_{\varphi}(x)\right\} .
$$

Hence $\partial^{*} \varphi(x) \equiv \mathbb{R}^{n}$ if $x$ is a minimizer of $\varphi$ over $\mathbb{R}^{n}$, and if $\varphi$ is continuous on $\mathbb{R}^{n}$ then $\partial^{*} \varphi(x)$ is the normal cone of $\bar{L}_{\varphi}(x)$, that is

$$
\partial^{*} \varphi(x)=N\left(\bar{L}_{\varphi}(x), x\right):=\left\{g \in \mathbb{R}^{n}:\langle g, y-x\rangle \leq 0 \forall y \in \bar{L}_{\varphi}(x)\right\} .
$$

Furthermore $\partial^{*} \varphi(x)$ contains nonzero vector [8]. This subdifferential thus is also called normal-subdifferential.

Lemma 2.2. ([11], [21]) Assume that $\varphi: \mathbb{R}^{n} \rightarrow \mathbb{R}$ is continuous and quasiconvex. Then

$$
\begin{gathered}
\partial^{*} \varphi(x) \neq \emptyset \quad \forall x \in \mathbb{R}^{n}, \\
0 \in \partial^{*} \varphi(x) \Leftrightarrow x \in \operatorname{argmin}\left\{\varphi(y): y \in \mathbb{R}^{n}\right\} .
\end{gathered}
$$

For simplicity of notation, let $f_{k}(x):=f\left(x^{k}, x\right)$. For the star-subdifferential we have the following results will be used in the sequel.

Lemma 2.3. [11] If $B(\bar{x}, \epsilon) \subset L_{f_{k}}\left(x^{k}\right)$ for some $\bar{x} \in \mathbb{R}^{n}$ and $\epsilon \geq 0$, then

$$
\left\langle g^{k}, x^{k}-\bar{x}\right\rangle>\epsilon \text {. }
$$


Lemma 2.4. Let $h: \mathbb{R}^{n} \longrightarrow \mathbb{R}$ be a quasiconvex L-Lipschitz continuous function on $\mathbb{R}^{n}$. Then for every $y \in \bar{L}_{h}(z), g \in N\left(\bar{L}_{h}(z), z\right)$ such that $\|g\|=1$, it holds

$$
L\langle g, y-z\rangle \leq h(y)-h(z) \leq 0,
$$

Proof. Let $y \in \bar{L}_{h}(z)$. Thanks to the continuity of the function $h$, we have $h(y)-h(z) \leq 0$. Since $z \notin L_{h}(z), N\left(\bar{L}_{h}(z), z\right) \neq \emptyset$. Take $g \in N\left(\bar{L}_{h}(z), z\right)$ such that $\|g\|=1$, then

$$
\langle g, x-z\rangle \leq 0 \quad \forall x \in \bar{L}_{h}(z)
$$

Let $H(g)$ be the supporting hyperplane of $\bar{L}_{h}(z)$ at $z$, that is defined by

$$
H(g)=\left\{x \in \mathbb{R}^{n} \mid \quad\langle g, x-z\rangle=0\right\} .
$$

Since $L_{h}(z)$ is open, $H(g) \cap L_{h}(z)=\emptyset$. If $y \in H(g)$ then it is clear that (5) is true. If $y \notin H(g)$, let $P(y)$ be the projection of $y$ onto the hyperplane $H(g)$. Then $h(P(y)) \geq h(z)$.

On one hand,

$$
\begin{aligned}
0 \leq h(z)-h(y) & \leq h(P(y))-h(y) \\
& \leq L\|P(y)-y\| .
\end{aligned}
$$

On the other hand,

$$
\begin{aligned}
\|P(y)-y\| & =\langle g, P(y)-y\rangle \\
& =\langle g, P(y)-z\rangle+\langle g, z-y\rangle \\
& =\langle g, z-y\rangle \text { (because } P(y) \in H(g))
\end{aligned}
$$

From (6) and (7),

$$
L\langle g, y-z\rangle \leq h(y)-h(z) \leq 0
$$

We also need the following result to find the subdifferential in some important cases. Some calculus rules, optimality conditions and minimization methods concerning these subdifferentials have been studied in [5, 8, 9, 10, 11, 13, 20, 21.

Lemma 2.5. ([11]) Suppose $\varphi(x)=a(x) / b(x)$ for all $x \in$ dom $\varphi$, where $a$ is a convex function, $b$ is finite and positive on dom $\varphi$, dom $\varphi$ is convex and one of the following conditions holds

(a) $b$ is affine;

(b) a is nonnegative on dom $\varphi$ and $b$ is concave;

(c) a is nonpositive on dom $\varphi$ and $b$ is convex.

Then $\varphi$ is quasiconvex and $\partial(a-\alpha b)(x)$ is a subset of $\partial^{*} \varphi(x)$ for $\alpha=\frac{a(x)}{b(x)}$. 


\section{A Parallel Algorithm and Its Convergence}

For presentation of the algorithm and its convergence, we make the following assumptions:

\section{Assumptions}

(A1) For every $x \in C$, the function $f(x,$.$) is continuous, quasiconvex on \mathbb{R}^{n}$, and $f(.,$.$) is upper semicontinuous on an open set containing C \times C$;

(A2) The bifunction $f$ is pseudomonotone on $C$, that is

$$
f(x, y) \geq 0 \Rightarrow f(y, x) \leq 0 \quad \forall x, y \in C,
$$

and paramonotone on $C$ with respect to $S(E P)$, that is

$$
x \in S(E P), y \in C \text { and } f(x, y)=f(y, x)=0 \Rightarrow y \in S(E P) .
$$

The paramonotonicity of a bifunction is an extension of that for an operator, see e.g., [10, which has been used in some papers, see, for example, [1, 22, 25,

(A3) The solution set $S(E P)$ is nonempty.

For simplicity of notation, for $\omega^{T}=\left(\omega_{1}, \ldots, \omega_{m}\right)$ with $\omega_{i}>0$ for every $i$ and $\sum_{i=1}^{m} \omega_{i}=1$, let us define the operator $P_{\omega}$ as $P_{\omega}(x):=\sum_{i=1}^{m} \omega_{i} P_{C_{i}}(x)$ for every $x$. It is easy to see that $P_{\omega}$ is nonexpansive for any $\omega$. Since $C \subseteq C_{i}$ for every $i$, it follows that $P_{C_{i}}(x)=P_{\omega}(x)=x$ for every $x \in C$. For this operator we have the following result.

Lemma 3.1. ([5], [6] ) If $0<\omega_{i}<1$ for all $i=1,2, \ldots, n$ and $\bar{x}$ is a fixed poind of $P_{\omega}$, that is $\bar{x}=P_{\omega}(\bar{x})$, then $\bar{x} \in C=\cap_{i=1}^{m} C_{i}$.

Now we are in a position to describe an algorithm for solving equilibrium $(E P)$, where the feasible domain $C:=\cap_{j=1}^{m} C_{j}$. The algorithm is a projectionsubgradient one that takes the projection independently on each component set. 
Algorithm 1 Subgradient-Projection Algorithm

Take real sequences $\left\{\alpha_{k}\right\},\left\{\lambda_{k}\right\}$ and positive numbers $\omega_{1}, \ldots, \omega_{m}$ satisfying the following conditions

$$
\begin{gathered}
\alpha_{k}>0 \quad \forall k \in \mathbb{N} \\
\sum_{k=1}^{\infty} \alpha_{k}=+\infty, \quad \sum_{k=1}^{\infty} \alpha_{k}^{2}<+\infty . \\
0<\underline{\lambda} \leq \lambda_{k} \leq \bar{\lambda}<1 \quad \forall k \in \mathbb{N}, \\
0<\omega_{i}<1 \quad \forall i=1, \ldots, m, \sum_{i=1}^{m} \omega_{i}=1
\end{gathered}
$$

Initial Step: choose $x^{0} \in \mathbb{R}^{n}$, let $k=0$.

Step k $(0,1 \ldots)$ : Having $x^{k} \in \mathbb{R}^{n}$, take

$$
g^{k} \in \partial_{2}^{*} f\left(x^{k}, x^{k}\right):=\left\{g \in \mathbb{R}^{n}:\left\langle g, y-x^{k}\right\rangle<0 \quad \forall y \in L_{f_{k}}\left(x^{k}\right)\right\} .
$$

If $g^{k}=0$ and $x^{k} \in C$, stop: $x^{k}$ is a solution.

If $g^{k} \neq 0$, normalize $g^{k}$ to obtain $\left\|g^{k}\right\|=1$.

Compute

$$
x^{k+1}=\left(1-\lambda_{k}\right) x^{k}+\lambda_{k} P_{\omega}\left(x^{k}-\alpha_{k} g^{k}\right)
$$

If $x^{k+1}=x^{k}$ and $x^{k} \in C$ then stop: $x^{k}$ is a solution.

Else update $k \longleftarrow k+1$.

The sequence of the iterates generated by the algorithm has the following properties:

Proposition 3.2. For every $z \in C=\cap_{i=1}^{m} C_{i}$, and $k \in \mathbb{N}$, the following inequality holds

$\left\|x^{k+1}-z\right\|^{2} \leq\left\|x^{k}-z\right\|^{2}+2 \lambda_{k} \alpha_{k}\left\langle g^{k}, z-x^{k}\right\rangle+\lambda_{k} \alpha_{k}^{2}-\lambda_{k}\left(1-\lambda_{k}\right)\left\|x^{k}-P_{\omega}\left(x^{k}-\alpha_{k} g^{k}\right)\right\|^{2}$.

Proof. Let $z \in C$, by using the elementary equality

$$
\|(1-t) a+t b\|^{2}=(1-t)\|a\|^{2}+t\|b\|^{2}-t(1-t)\|a-b\|^{2}
$$

with $a=x^{k}-z, b=P_{\omega}\left(x^{k}-\alpha_{k} g^{k}\right)-z, t=\lambda_{k}$, we have

$$
\begin{aligned}
\left\|x^{k+1}-z\right\|^{2}= & \left\|x^{k}+\lambda_{k}\left[P_{\omega}\left(x^{k}-\alpha_{k} g^{k}\right)-x^{k}\right]-z\right\|^{2} \\
= & \left(1-\lambda_{k}\right)\left\|x^{k}-z\right\|^{2}+\lambda_{k}\left\|P_{\omega}\left(x^{k}-\alpha_{k} g^{k}\right)-z\right\|^{2} \\
& -\lambda_{k}\left(1-\lambda_{k}\right)\left\|x^{k}-P_{\omega}\left(x^{k}-\alpha_{k} g^{k}\right)\right\|^{2} .
\end{aligned}
$$


In addition,

$$
\begin{aligned}
& \left\|P_{\omega}\left(x^{k}-\alpha_{k} g^{k}\right)-z\right\|^{2} \\
= & \left\|\sum_{i=1}^{m} \omega_{i}\left(P_{C_{i}}\left(x^{k}-\alpha_{k} g^{k}\right)-z\right)\right\|^{2} \\
\leq & \sum_{i=1}^{m} \omega_{i}\left\|P_{C_{i}}\left(x^{k}-\alpha_{k} g^{k}\right)-z\right\|^{2} \\
\leq & \sum_{i=1}^{m} \omega_{i}\left\|x^{k}-\alpha_{k} g^{k}-z\right\|^{2} \\
= & \left\|x^{k}-\alpha_{k} g^{k}-z\right\|^{2} \\
= & \left\|x^{k}-z\right\|^{2}-2 \alpha_{k}\left\langle g^{k}, x^{k}-z\right\rangle+\alpha_{k}^{2} .
\end{aligned}
$$

From (10) and the last equality, it follows that

$$
\left\|x^{k+1}-z\right\|^{2} \leq\left\|x^{k}-z\right\|^{2}+2 \lambda_{k} \alpha_{k}\left\langle g^{k}, z-x^{k}\right\rangle+\lambda_{k} \alpha_{k}^{2}-\lambda_{k}\left(1-\lambda_{k}\right)\left\|x^{k}-P_{\omega}\left(x^{k}-\alpha_{k} g^{k}\right)\right\|^{2} .
$$

Lemma 3.3.

$$
\liminf _{k \rightarrow+\infty}\left\langle g^{k}, x^{k}-z\right\rangle \leq 0, \forall z \in C .
$$

Proof. From Proposition 3.2 and $0<\underline{\lambda} \leq \lambda_{k} \leq \bar{\lambda}<1$, we obtain

$$
2 \alpha_{k}\left\langle g^{k}, x^{k}-z\right\rangle \leq \frac{1}{\underline{\lambda}}\left(\left\|x^{k}-z\right\|^{2}-\left\|x^{k+1}-z\right\|^{2}\right)+\alpha_{k}^{2} .
$$

Applying this inequality for every $k=1, \ldots \infty$, and summing up we obtain

$$
\sum_{k=1}^{\infty} \alpha_{k}\left\langle g^{k}, x^{k}-z\right\rangle<+\infty
$$

which together with $\sum_{k=1}^{\infty} \alpha_{k}=+\infty$, implies

$$
\liminf _{k \rightarrow+\infty}\left\langle g^{k}, x^{k}-z\right\rangle \leq 0 \text {. }
$$

We have the following convergence result.

Theorem 3.4. Under the assumptions (A1)- (A3) it holds that

(i) If Algorithm 3 terminates at iteration $k$, then $x^{k}$ is a solution of $(E P)$;

(ii) If the algorithm does not terminate, then there exists a subsequence of $\left\{x^{k}\right\}$ converges to a solution of $(E P)$ whenever $\left\{x^{k}\right\}$ is bounded. In addition, if Problem $(E P)$ is uniquely solvable, in particular, $f$ is strongly monotone, the whole sequence $\left\{x^{k}\right\}$ converges to the solution. 
Proof. (i) Suppose that the algorithm terminates at iteration $k$. Then, if $0 \in$ $\partial_{2}^{*} f\left(x^{k}, x^{k}\right)$ and $x^{k} \in C$, we have

$$
x^{k} \in \operatorname{argmin}_{y \in \mathbb{R}^{n}} f\left(x^{k}, y\right) .
$$

Hence, $f\left(x^{k}, y\right) \geq f\left(x^{k}, x^{k}\right)=0$ for every $y \in \mathbb{R}^{n}$. Since $x^{k} \in C$, it is a solution of $(E P)$.

If $x^{k+1}=x^{k}$ and $x^{k} \in C$, we have

$$
\begin{aligned}
P_{\omega}\left(x^{k}-\alpha_{k} g^{k}\right) & =x^{k} . \\
\Longleftrightarrow \sum_{i=1}^{m} \omega_{i} P_{C_{i}}\left(x^{k}-\alpha_{k} g^{k}\right) & =x^{k} .
\end{aligned}
$$

For every $y \in C \subseteq C_{i}$ and every $i \in\{1, \ldots, m\}$, it holds that

$$
\left\langle x^{k}-\alpha_{k} g^{k}-P_{C_{i}}\left(x^{k}-\alpha_{k} g^{k}\right), y-P_{C_{i}}\left(x^{k}-\alpha_{k} g^{k}\right)\right\rangle \leq 0 .
$$

Equivalently

$$
\left\langle x^{k}-P_{C_{i}}\left(x^{k}-\alpha_{k} g^{k}\right), y-P_{C_{i}}\left(x^{k}-\alpha_{k} g^{k}\right)\right\rangle \leq\left\langle\alpha_{k} g^{k}, y-P_{C_{i}}\left(x^{k}-\alpha_{k} g^{k}\right)\right\rangle .
$$

Multiplying by $\omega_{i}$ and summing up we obtain

$$
\sum_{i=1}^{m} \omega_{i}\left\langle x^{k}-P_{C_{i}}\left(x^{k}-\alpha_{k} g^{k}\right), y-P_{C_{i}}\left(x^{k}-\alpha_{k} g^{k}\right)\right\rangle \leq \sum_{i=1}^{m} \omega_{i}\left\langle\alpha_{k} g^{k}, y-P_{C_{i}}\left(x^{k}-\alpha_{k} g^{k}\right)\right\rangle .
$$

By a simple computation, using $\sum_{i=1}^{m} \omega_{i} P_{C_{i}}\left(x^{k}-\alpha_{k} g^{k}\right)=x^{k}$ and $\sum_{i=1}^{m} \omega_{i}=$ 1 , we arrive at

$$
-\left\|x^{k}\right\|^{2}+\left\|x^{k}\right\|^{2} \leq \sum_{i=1}^{m} \omega_{i} \alpha_{k}\left\langle g^{k}, y-P_{C_{i}}\left(x^{k}-\alpha_{k} g^{k}\right)\right\rangle=\alpha_{k}\left\langle g^{k}, y-x^{k}\right\rangle .
$$

Since $g^{k} \in \partial^{*} f\left(x^{k}, x^{k}\right)$, the last inequality $\left\langle g^{k}, y-x^{k}\right\rangle \geq 0, \forall y \in C$ implies $f\left(x^{k}, y\right) \geq f\left(x^{k}, x^{k}\right)=0$ for every $y \in C$, which means that $x^{k}$ is a solution of (EP).

(ii) We consider two cases.

Case 1: There exists a solution $x^{*} \in S(E P)$ and an index $k_{0}$ such that for $k \geq k_{0}$,

$$
\left\|x^{k+1}-x^{*}\right\| \leq\left\|x^{k}-x^{*}\right\| .
$$

By the nonnegativity of $\left\|x^{k}-x^{*}\right\|$, we conclude that the sequence $\left\{\left\|x^{k}-x^{*}\right\|\right\}$ is convergent.

Moreover, from Proposition 3.2 it follows that

$$
\begin{aligned}
& \lambda_{k}\left(1-\lambda_{k}\right)\left\|x^{k}-P_{\omega}\left(x^{k}-\alpha_{k} g^{k}\right)\right\|^{2} \\
\leq & \left\|x^{k}-x^{*}\right\|^{2}-\left\|x^{k+1}-x^{*}\right\|^{2}-2 \lambda_{k} \alpha_{k}\left\langle g^{k}, x^{k}-x^{*}\right\rangle+\lambda_{k} \alpha_{k}^{2} .
\end{aligned}
$$


When $k$ goes to infinity, the right hand side of the above inequality goes to 0 . Since $0<\underline{\lambda} \leq \lambda_{k} \leq \bar{\lambda}<1$, we obtain

$$
\lim _{k \rightarrow \infty}\left\|x^{k}-P_{\omega}\left(x^{k}-\alpha_{k} g^{k}\right)\right\|=0 .
$$

We also have that

$$
\begin{aligned}
\left\|x^{k}-P_{\omega}\left(x^{k}\right)\right\| & \leq\left\|x^{k}-P_{\omega}\left(x^{k}-\alpha_{k} g^{k}\right)\right\|+\left\|P_{\omega}\left(x^{k}-\alpha_{k} g^{k}\right)-P_{\omega}\left(x^{k}\right)\right\| \\
& \leq\left\|x^{k}-P_{\omega}\left(x^{k}-\alpha_{k} g^{k}\right)\right\|+\left\|x^{k}-\alpha_{k} g^{k}-x^{k}\right\| \\
& \leq\left\|x^{k}-P_{\omega}\left(x^{k}-\alpha_{k} g^{k}\right)\right\|+\alpha_{k} .
\end{aligned}
$$

Since $\lim _{k \rightarrow \infty} \alpha_{k}=0$,

$$
\lim _{k \rightarrow \infty}\left\|x^{k}-P_{\omega}\left(x^{k}\right)\right\|=0 .
$$

From Lemma 3.3 follows

$$
\liminf _{k \rightarrow \infty}\left\langle g^{k}, x^{k}-x^{*}\right\rangle \leq 0 .
$$

Let $\left\{x^{k_{i}}\right\}$ be a subsequence of $\left\{x^{k}\right\}$ such that

$$
\lim _{i \rightarrow \infty}\left\langle g^{k_{i}}, x^{k_{i}}-x^{*}\right\rangle=\liminf _{k \rightarrow \infty}\left\langle g^{k}, x^{k}-z\right\rangle .
$$

Since $\left\{x^{k}\right\}$ is bounded, $\left\{x^{k_{i}}\right\}$ is bounded too. Let $\bar{x}$ be a limit point of $\left\{x^{k_{i}}\right\}$, and without loss of generality we assume that

$$
\lim _{i \rightarrow \infty} x^{k_{i}}=\bar{x} .
$$

It is clear that

$$
\begin{aligned}
\| x^{k_{i}}-P_{\omega}(\bar{x} \| & \leq\left\|x^{k_{i}}-P_{\omega}\left(x^{k_{i}}\right)\right\|+\left\|P_{\omega}(\bar{x})-P_{\omega}\left(x^{k_{i}}\right)\right\| \\
& \leq\left\|x^{k_{i}}-P_{\omega}\left(x^{k_{i}}\right)\right\|+\left\|\bar{x}-x^{k_{i}}\right\| .
\end{aligned}
$$

Thanks to (13) and (15), we have

$$
\lim _{i \rightarrow \infty} x^{k_{i}}=P_{\omega}(\bar{x}) .
$$

Combining this with (15), we see that $P_{\omega}(\bar{x})=\bar{x}$, which together with $\omega_{i}>0$ for every $i$ implies $\bar{x} \in C$.

In addition, since $x^{*}$ is a solution, by pseudomonotonicity of $f$ on $C$, we have $f\left(\bar{x}, x^{*}\right) \leq 0$. We show that $f\left(\bar{x}, x^{*}\right)=0$. In fact, by contradiction, we assume that there exists $a>0$ such that

$$
f\left(\bar{x}, x^{*}\right) \leq-a .
$$

Since $f(.,$.$) is upper semicontinuous on an open set containing C \times C$, there exist positive numbers $\epsilon_{1}, \epsilon_{2}$ such that, for any $x \in B\left(\bar{x}, \epsilon_{1}\right), y \in B\left(x^{*}, \epsilon_{2}\right)$ we have $f(x, y) \leq-\frac{a}{2}$. 
On the other hand, $\lim _{i \rightarrow \infty} x^{k_{i}}=\bar{x}$ implies that there exist $i_{0}$ such that for $i \geq i_{0}, x^{k_{i}}$ belongs to $B\left(\bar{x}, \epsilon_{1}\right)$. So, for $i \geq i_{0}$ and $y \in B\left(x^{*}, \epsilon_{2}\right)$, we have

$$
f\left(x^{k_{i}}, y\right) \leq-\frac{a}{2},
$$

which implies that $B\left(x^{*}, \epsilon_{2}\right) \subset L_{f_{k_{i}}}\left(x^{k_{i}}\right)$. In addition, $g^{k_{i}} \neq 0$, because if $g^{k_{i}}=0$ then $f\left(x^{k_{i}}, y\right) \geq f\left(x^{k_{i}}, x^{k_{i}}\right)=0$ for every $y \in \mathbb{R}^{n}$, which contradicts to (18). By Lemma 2.3, for $i \geq i_{0}$, it holds that

$$
\left\langle g^{k_{i}}, x^{k_{i}}-x^{*}\right\rangle>\epsilon_{2},
$$

which contradicts to (14). Thus, $\bar{x} \in C$ and $f\left(\bar{x}, x^{*}\right)=0$. Again by pseudomonotonicity, we obtain $f\left(x^{*}, \bar{x}\right)=0$. Then, from paramonotonicity of $f$ it follows that $\bar{x}$ is a solution of $(E P)$. In addition, since $\left\{\left\|x^{k}-x^{*}\right\|\right\}$ is convergent for every solution $x^{*}$ of $(E P)$, the sequence $\left\{x^{k}\right\}$ converges to $\bar{x}$ which is a solution of $(E P)$.

Case 2: For any solution $x^{*}$ of (EP) there exists a subsequence $\left\{x_{k_{i}}\right\}$ of $\left\{x^{k}\right\}$ that satisfies

$$
\left\|x^{k_{i}}-x^{*}\right\|<\left\|x^{k_{i}+1}-x^{*}\right\| .
$$

Now again, by Proposition 3.2 applying with $x^{*}$, we can write

$$
\begin{aligned}
& \lambda_{k_{i}}\left(1-\lambda_{k_{i}}\right)\left\|x^{k_{i}}-P_{\omega}\left(x^{k_{i}}-\alpha_{k_{i}} g^{k_{i}}\right)\right\|^{2} \\
\leq & \left\|x^{k_{i}}-x^{*}\right\|^{2}-\left\|x^{k_{i}+1}-x^{*}\right\|^{2}-2 \lambda_{k_{i}} \alpha_{k_{i}}\left\langle g^{k_{i}}, x^{k_{i}}-x^{*}\right\rangle+\lambda_{k_{i}} \alpha_{k_{i}}^{2} . \\
\leq & -2 \lambda_{k_{i}} \alpha_{k_{i}}\left\langle g^{k_{i}}, x^{k_{i}}-x^{*}\right\rangle+\lambda_{k_{i}} \alpha_{k_{i}}^{2} .
\end{aligned}
$$

Since $\left\{x^{k_{i}}\right\}$ is bounded, and $0<\lambda_{k}<1, \alpha_{k} \rightarrow 0$, from the last inequality it follows that

$$
\lim _{i \rightarrow \infty}\left\|x^{k_{i}}-P_{\omega}\left(x^{k_{i}}-\alpha_{k_{i}} g^{k_{i}}\right)\right\|=0 .
$$

Also from (19) follows

$$
\begin{gathered}
\left\langle g^{k_{i}}, x^{k_{i}}-x^{*}\right\rangle \leq \frac{\alpha_{k_{i}}}{2} . \\
\limsup _{i \rightarrow \infty}\left\langle g^{k_{i}}, x^{k_{i}}-x^{*}\right\rangle \leq 0 .
\end{gathered}
$$

Now by the same argument as in Case 1, we see that every limit point of the sequence $\left\{x_{k_{i}}\right\}$ belongs to the solution set $S(E P)$.

Suppose now that the solution is unique, then the sequence $\left\{x^{k}\right\}$ converges to the unique solution $x^{*}$ of $(E P)$. In fact, without loss of generality, we may assume that for $k \notin\left\{k_{j}\right\}$. Then

$$
\left\|x^{k+1}-x^{*}\right\| \leq\left\|x^{k}-x^{*}\right\| .
$$

For any $\epsilon>0$ there exist $i_{0}$ such that for $i \geq i_{0}$,

$$
\left\|x^{k_{i}}-x^{*}\right\|<\epsilon .
$$


Furthermore, for any $k>k_{i_{0}}$ that does not belong to $\left\{k_{i}\right\}$, there exists $i_{1} \geq i_{0}$ such that $k_{i_{1}}<k<k_{i_{1}+1}$. Then $\left\|x^{k}-x^{*}\right\| \leq\left\|x^{k_{i_{1}}}-x^{*}\right\|<\epsilon$, which means that $\left\|x^{k}-x^{*}\right\|<\epsilon$ for $k \geq k_{i_{0}}$. Hence, the whole sequence $\left\{x^{k}\right\}$ converges to the unique solution of $(E P)$.

Remark. The assumption on boundedness of the sequence $\left\{x^{k}\right\}$ is ensured if either

(C1) The set $C_{\omega}:=\left\{x \in \mathbb{R}^{n}: x=\sum_{j=1}^{m} \omega_{j} x^{j}: x^{j} \in C_{j} j=1, \ldots, m\right\}$ is bounded, and $x^{0} \in C_{\omega}$,

or

(C2) The bifuntion $f(x, y)$ is pseudomonotone on $C_{\omega}$ and for each $x$, the function $f_{x}():.=f(x,$.$) is Lipschitz continuous with constant L_{x}$, and

$$
C \cap S\left(C_{\omega}, f\right) \neq \emptyset
$$

where $S\left(C_{\omega}, f\right)$ stands for the solution-set of the equilibrium problem

$$
\text { find } \bar{z} \in C_{\omega}: f(\bar{z}, z) \geq 0 \forall z \in C_{\omega} \text {. }
$$

Indeed, according to the algorithm, $x^{k+1}$ is a convex combination of elements of the convex set $C_{\omega}$, we see that $x^{k+1} \in C_{\omega}$ for every $k=0,1, \ldots$, , with implies that $\left\{x^{k}\right\}$ is bounded.

To see the assertion for (C2), we apply Lemma 2.4 with $h(x):=f\left(x^{k}, x\right), z:=x^{k}$ and $y=\bar{z} \in C \cap S\left(C_{\omega}, f\right)$. Then $f\left(y, x^{k}\right) \geq 0$, which, by pseudomonotonicity, implies $f\left(x^{k}, y\right) \leq 0$ for all $k$. Hence $y \in \bar{L}_{h}\left(x^{k}\right)$. Thus by Lemma 2.4 $\left\langle g^{k}, \bar{z}-\right.$ $\left.x^{k}\right\rangle \leq 0$. Then, by applying Proposition 3.2 with $z=\bar{z}$, we obtain

$$
\left\|x^{k+1}-\bar{z}\right\|^{2} \leq\left\|x^{k}-\bar{z}\right\|^{2}+\alpha_{k}^{2}
$$

Hence, $\left\{\left\|x^{k}-\bar{z}\right\|^{2}\right\}$ is convergence, and therefore $\left\{x^{k}\right\}$ is bounded

In the case of optimization problem, where $f(x, y):=\varphi(y)-\varphi(x)$, Condition (C2) means that $\varphi$ is Lipschitz continuous and there exists $\bar{z} \in C$ such that $\bar{z}$ is a minimizer of the function $\varphi(y)$ on $C_{\omega}$.

Condition $(C 2)$ is inspired by the paper [23], where the equilibrium problems of the form

$$
\text { Find } x^{*} \in P \cap Q: f\left(x^{*}, y\right) \geq 0 \forall y \in Q,
$$

with $P, Q$ being convex sets have been studied. This common solution problem has been attracted much attention of researchers in recent years.

\section{Computational Experience}

To test the algorithm, we consider the equilibrium problem

$$
\text { Find } x^{*} \in C \text { such that } f\left(x^{*}, y\right) \geq 0 \quad \forall y \in C,
$$


where the bifunction $f(x, y)$ is defined by

$$
f(x, y)=\left\langle A x+b, \frac{A_{1} y+b_{1}}{c^{T} y+d}-\frac{A_{1} x+b_{1}}{c^{T} x+d}\right\rangle,
$$

with $A, A_{1} \in \mathbb{R}^{n \times n}, b, b_{1}, c \in \mathbb{R}^{n}, d \in \mathbb{R}$ and $C \subset\left\{x \mid \quad c^{T} x+d>0\right\}$.

By applying Proposition 4.1 in [10] to the differentiable function $g(x):=$ $A^{T}\left(\frac{A_{1} x+b_{1}}{c^{T} x+d}\right)$ one can easily check that $f$ is monotone on $C$ if and only if the matrix

$$
\hat{A}_{1}(x)=A^{T}\left[A_{1} c^{T} x-A_{1} x c^{T}\right]+A^{T}\left[A_{1} d-b_{1} c^{T}\right]
$$

is positive semidefinite and paramonotone if $\hat{A}_{1}(x)$ is symmetric for any $x \in C$.

We test the algorithm with the following three examples, where the projection onto each component set has a closed form. For each problem we chose $\lambda_{k}=1 / 2$ for every $k$, and $\omega_{i}=1 / m$ for all $i$. We stop the computation at iteration $k$ if $g^{k}=0$ or err $1:=\left\|x^{k}-x^{k+1}\right\|<10^{-4}$ and err $2=$ $\left\|x^{k}-P_{C_{1}}\left(x^{k}\right)\right\|+\ldots+\left\|x^{k}-P_{C_{m}}\left(x^{k}\right)\right\|<10^{-1}$, or the number of iteration exceeds 1000 .

The average time and average errors for each size are reported in Tables 1 , 2, 3 with different sizes, a hundred of problems have been tested for each size.

Example 4.1. In this example, we take

$$
C=C_{1} \cap C_{2},
$$

where $C_{1}=[1,3]^{n}$ and $C_{2}=\left\{x \in \mathbb{R}^{n} \mid \quad\|x\| \leq 3\right\}$. Each entries of $A, A_{1}, b, b_{1}, c, d$ is uniformly generated in the interval $[0,1]$.

Table 1: Algorithm with $\alpha_{k}=\frac{100}{k+1}$

\begin{tabular}{ccccc}
\hline \hline $\mathrm{n}$ & N. of prob. & CPU-times(s) & Error 1 & Error 2 \\
\hline 5 & 100 & 5.674775 & 0.000127 & 0.188295 \\
10 & 100 & 6.990620 & 0.000092 & 0.187407 \\
20 & 100 & 8.7481101 & 0.000085 & 0.186929 \\
50 & 100 & 40.770369 & 0.000083 & 0.186708 \\
\hline
\end{tabular}

Example 4.2. In this example, we take

$$
C=C_{1} \cap C_{2} \cap C_{3},
$$

where $C_{1}=[1,3]^{n}, C_{2}=\left\{x \in \mathbb{R}^{n} \mid \quad\|x\| \leq 3\right\}$ and $C_{3}=\left\{x \in \mathbb{R}^{n} \mid \quad \sum_{i=1}^{n} x_{i} \geq\right.$ $N+1\}$. Each entries of $A, A_{1}, b, b_{1}, c, d$ is uniformly generated in the interval $[0,1]$.

Example 4.3. In this example, we take

$$
C=C_{1} \cap C_{2}
$$


Table 2: Algorithm with $\alpha_{k}=\frac{100}{k+1}$

\begin{tabular}{ccccc}
\hline \hline $\mathrm{n}$ & N. of prob. & CPU-times(s) & Error 1 & Error 2 \\
\hline 5 & 100 & 11.339168 & 0.000224 & 0.383520 \\
10 & 100 & 11.638716 & 0.000182 & 0.383298 \\
20 & 100 & 13.612659 & 0.000170 & 0.386529 \\
50 & 100 & 42.999559 & 0.000165 & 0.388552 \\
\hline
\end{tabular}

where $C_{1}=[1,3]^{n}$ and $C_{2}=\left\{x \in \mathbb{R}^{n} \mid \quad \sum_{i=1}^{3} x_{i} \geq 3\right\}$. Each entries of $A, A_{1}, b, b_{1}, c, d$ is uniformly generated in the interval $[0,1]$.

Note that $x^{*} \in C$ is a solution of the equilibrium problem (EP) if and only if $x^{*}$ belongs to the solution set of the following affine fractional programming problem

$$
\min _{y \in C} g\left(x^{*}, y\right),
$$

where $g(x, y)=\left\langle A x+b, \frac{A_{1} y+b_{1}}{c^{T} y+d}\right\rangle$. Thus we can use linear programming algorithms to compute

$$
\operatorname{err}_{3}=\frac{-\min _{y \in C} g\left(P_{C}\left(x^{k}\right), y\right)+g\left(P_{C}\left(x^{k}\right), P_{C}\left(x^{k}\right)\right)}{g\left(P_{C}\left(x^{k}\right), P_{C}\left(x^{k}\right)\right)} .
$$

and use it as a stopping criterion.

Table 3: Algorithm with $\alpha_{k}=\frac{100}{k+1}$

\begin{tabular}{cccccc}
\hline \hline $\mathrm{n}$ & N. of prob. & CPU-times(s) & Error1 & Error 2 & Error 3 \\
\hline 5 & 100 & 10.218638 & 0.000210 & 0.199647 & 0.058665 \\
10 & 100 & 10.811854 & 0.000275 & 0.200397 & 0.051829 \\
20 & 100 & 12.970668 & 0.000498 & 0.200389 & 0.074603 \\
50 & 100 & 45.202791 & 0.000731 & 0.200382 & 0.086886 \\
\hline
\end{tabular}

\section{Conclustion}

We have proposed an iterative star-subgradient projection algorithm for solving a class of equilibrium problems over the intersection of closed, convex sets, where the bifunction is quasiconvex in its second variable. The search direction at each iteration is defined by a star-subgradient at the current iterate, and the projection is executed independently on each component of the intersection sets. Convergence of the algorithm has been shown, and some illustrative examples have been solved. 


\section{References}

[1] P. N. Anh, L. D. Muu: A hybrid subgradient algorithm for nonexpansive mapping and equilibrium problems, Optim. Lett. 8, 727-738 (2014).

[2] G. Bigi , M. Castellani, M. Pappalardo, M. Passacantando: Existence and solution methods for equilibria. Eur. Oper. Res. 227, 1-11 (2013).

[3] G. Bigi, , M. Castellani, M. Pappalardo, M. Passacantando: Nonlinear Programming Techniques for Equilibria Springer (2019).

[4] E. Blum, W. Oettli: From optimization and variational inequalities to equilibrium problems, Math. Student 62, 127-169 (1994).

[5] F. Deutsch, I. Yamada: Minimizing certain convex functions over the intersection of the fixed point sets of nonexpansive mappings. Numer. Funct. Anal, Optim. 19,33-56 (1998).

[6] P. M. Duc, Le D. Muu: A splitting algorithm for a class of bilevel equilibrium problems involving nonexpansive mappings, Optimization 65, 1855-1866 (2016).

[7] K. Fan: A minimax inequality and applications. In: Shisha O. (Ed.): Inequalities. Academic Press, New York, 103-113 (1972).

[8] H.P. Greenberg and W.P. Pierskalla: Quasi-conjugate functions and surogate duality, Cahiers Centre tudes Recherche Oper., 15, 437- 448, (1973).

[9] J. Gromicho: Quasiconvex Optimization and Location Theory, Kluwer Academic Publishers, Dordrecht, The Netherlands (1998).

[10] A.N. Iusem: On some properties of paramonotone operator, Convex Anal. 5, 269-278 (1998).

[11] K. C. Kiwiel: Convergence and efficiency of subgradient methods for quasiconvex minimization, Math. Program., Ser. A 90, 1-25 (2001).

[12] D. V. Hieu, P. K. Quy, L. V. Vy: Explicit iterative algorithms for solving equilibrium problems, Calcolo 56:11 https://doi.org/10.1007/s10092-0190308-5 (2019).

[13] I. Konnov: On convergent properties of a subgradient method, Optim. Meth. Software, 17 , 53-62 (2003).

[14] O. Mangasarian: Nonlinear Programming, McGraw-Hill, Newyork (1969).

[15] L. D. Muu, Oettli W.: Convergence of an adaptive penalty scheme for finding constrained equilibria, Nonlinear Anal.: TMA 18, 1159- 1166 (1992).

[16] L. D. Muu, T. D. Quoc: Regularization algorithms for solving monotone Ky Fan inequalities with application to a Nash-Cournot equilibrium model, J. Optim. Theory Appl. 142, 185-204 (2009). 
[17] Nikaido H., Isoda K.: Note on noncooperative convex games, Pacific of Math. 5, 807-815(1955).

[18] N. Nimanaa, A. P. Farajzadehb, N. Petrot: Adaptive subgradient method for the split quasiconvex feasibility problems, Optimization 65, 1885-1898 (2016).

[19] T. D. Quoc, Le D. Muu, V. H. Nguyen: Extragradient algorithms extended to equilibrium problems, Optimization 57, 749-776 (2008).

[20] J.-P. Penot: Are generalized derivatives useful for generalized convex functions? in: J.-P. Crouzeix, J.E. Martinez-Legaz and M. Volle (Ed.), Generalized convexity, Generalized Monotonicity: Recent Results,Kluwer Academic Publishers, Dordrecht, The Netherlands, 3-59 (1998).

[21] J.-P. Penot and C. Zalinescu: Elements of quasiconvex subdifferential calculus, J. Convex Anal., 7, 243-269 (2000).

[22] P. Santos, S. Scheimberg: An inexact subgradient algorithm for equilibrium problems, Comput. Appl. Math. 30, 91-107 (2011).

[23] P. Santos, S. Scheimberg: A modied projection algorithmfor constrained equilibrium problems, Optimization 66, 2051-2062 (2017).

[24] L. Q. Thuy, T. N. Hai: A projected subgradient algorithm for bilevel equilibrium problems and applications, J. Optim. Theory Appl. 175, 411-431 (2017).

[25] L. H. Yen, N. T. T. Huyen, L. D. Muu: A subgradient algorithmfor a class of nonlinear split feasibility problems: application to jointly constrained Nash equilibrium models, J. Glob. Optim. 73, 849-858 (2019).

[26] L. H. Yen, L. D. Muu: A subgradient method for equilibrium problems involving quasiconvex bifunction. https://arxiv.org/abs/1911.00181. 